\title{
Slow photons in vacuum as elementary particles
}

\section{Chander Mohan Singal}

Chander Mohan Singal, "Slow photons in vacuum as elementary particles," Proc. SPIE 9664, Ninth International Topical Meeting on Education and Training in Optics and Photonics, 96642T (24 October 2005); doi: $10.1117 / 12.2207784$

SDIE Event: Ninth International Topical Meeting on Education and Training in Optics and Photonics, 2005, Marseille, France 


\title{
Ref ETOP098
}

\section{Slow Photons in Vacuum as Elementary Particles}

\author{
Chander Mohan Singal
}

Department of Physics, Indian Institute of Technology-Delhi, Hauz Khas, New Delhi-110016, INDIA . E-Mail: drcmsingal@yahoo.com

\section{Abstract}

Maxwell equations for Electro-magnetic(EM) vector fields $\mathbf{E}$ and $\mathbf{B}$ are solved in vacuum, free from charges and currents, and EM wave packets propagating in $z$ direction are formed using cylindrical coordinates $(\mathrm{s}, \varphi, \mathrm{z})$ with an average propagation vector $\mathrm{k}_{0}$ and a radial parameter $\gamma$ used for defining Bessel function $J_{n}(\gamma s)$ of n'th order. It is shown that these wave packets have a group velocity $\mathrm{v}_{\mathrm{g}}=\mathrm{ck}_{0} /\left[\mathrm{k}_{0}{ }^{2}+\gamma^{2}\right]^{0.5}$ smaller than the standard velocity $\mathrm{c}$ of light in vacuum, and they spread in $z$ direction with progression of time, like particles having non-zero rest mass $m_{0}=\hbar \mathrm{y} / \mathrm{c}$. It is shown that these slow photons can describe motion of elementary particles like electrons and protons with regard to their velocity and linear momentum. Quantization of energy $U$ of these EM wave packets is done using the condition $U=\hbar c\left[k_{0}^{2}+\gamma^{2}+\sigma^{2}\right]^{0.5}$ and then the angular momentum determined for them, (here $\sigma$ is the standard deviation in the propagation vector $k$ ). After quantization, the $z$ components of the linear momentum and angular momentum of the wave packets are found to be $\hbar k_{0}$ and $n \hbar$, respectively. It is shown that for $y$ much smaller than $k_{0}$ these wave packets can appear like light photons, and for $\mathrm{y}$ much larger than $\mathrm{k}_{0}$ these wave packets can appear like electrons and protons, with regard to their mechanics .

\section{Key Words}

Slow Photon, Electron, Rest Mass, Energy, Velocity, Linear Momentum , Angular Momentum.

\section{Mathematical Formulation:}

\section{Summary}

Electro-magnetic (EM) fields $\mathbf{E}$ and $\mathbf{B}$ are well known ${ }^{1}$ to follow Maxwell equations giving divergence and curl of the vectors $\mathbf{E}$ and $\mathbf{B}$. Plane wave solutions are normally obtained by solving these equations in Cartesian coordinates, and velocity of these plane waves in vacuum is known to be $c=2.99792458 \times 10^{10}$ centimeter/second . However, the equations for fields $\mathbf{E}$ and $\mathbf{B}$ can be solved in cylindrical coordinates ( $\mathrm{s}, \varphi, z)$ as well, using functions of the type $J_{n}(\gamma s) \exp (i k z+i n \varphi-i \omega t)$ where $n$ is the order of the Bessel function $J_{n}$ with radial coordinate $s$ and Bessel parameter $\gamma, k$ is the wave propagation vector in $z$ direction, and $\omega$ is the angular frequency given by

$(\omega / c)^{2}=k^{2}+y^{2}$. In the present study wave packets have been formed in vacuum ( free from charges and currents ) using these solutions, and the time dependent motion of these wave packets is studied numerically with the help of a high speed computer. In the following analysis the Gaussian system of units is utilized for the EM fields. Maxwell equations ${ }^{1}$ for $\mathbf{E}$ and $\mathbf{B}$ fields in vacuum, free of charges and currents, are :

$\nabla \cdot \mathbf{E}=0 ; \quad \nabla \cdot \mathbf{B}=0 ; \quad \nabla \mathbf{x E}=-(\partial \mathbf{B} / \partial \mathrm{t}) / \mathrm{c} ; \quad \nabla \mathbf{x B}=(\partial \mathbf{E} / \partial \mathrm{t}) / \mathrm{c}$. (1) Each Cartesian component $\psi$ of $\mathbf{E}$ and $\mathbf{B}$ satisfies the wave equation :

$\nabla^{2} \psi=\left(\partial^{2} \psi / \partial \mathrm{t}^{2} \quad\right) \quad / \quad \mathrm{c}^{2}$

(2)

For the cylindrical coordinates, the $\mathbf{z}$ component of the $\mathbf{E}$ and $\mathbf{B}$ fields are the same as those for the Cartesian coordinates and thus for $E_{Z}$ or $B_{Z}$ the solution is taken from the above 
wave equation. The remaining components $E_{S}, E_{\phi}, B_{S}$ and $B_{\phi}$ are then evaluated directly from Maxwell equations. For transverse magnetic (TM) mode $B_{Z}$ is zero, and for transverse electric (TE) mode $E_{z}$ is zero. For TE mode, one set of real electric and magnetic field components in cylindrical coordinates is:

$(\mathrm{TE})_{1}$ :

$\mathrm{B}_{\mathrm{Z}}=\mathrm{A}(2 \pi \mathrm{R} / \gamma)^{-0.5} \quad J_{\mathrm{n}} \int \cos (\theta)[\mathrm{g}(\mathrm{k})]^{0.5} \mathrm{dk}$;

$B_{S}=-A(2 \pi R \gamma)^{-0.5}\left(\partial J_{n} / \partial s\right) \int[k / \gamma] \sin (\theta)[g(k)]^{0.5} d k ;$

$B_{\phi}=-A(2 \pi R \gamma)^{-0.5} \mathrm{n}\left(J_{\mathrm{n}} / \mathrm{s}\right) \int[\mathrm{k} / \gamma] \cos (\theta)[\mathrm{g}(\mathrm{k})]^{0.5} \mathrm{dk}$;

$E_{S}=-A(2 \pi R \gamma)^{-0.5} n\left(J_{n} / s\right) \int[\omega /(c \gamma)] \cos (\theta)[g(k)]^{0.5} d k$;

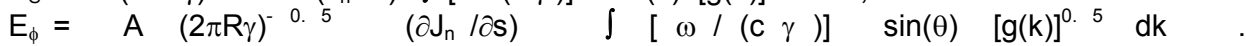

(3)

where $\theta=(k z+n \phi-\omega t)$, A is an amplitude factor, $\mathrm{R}$ is the large radius of the cylinder within which the Bessel function is normalized. For forming the wave packet, square root of the Gaussian function $\mathrm{g}(\mathrm{k})$ has been multiplied with each of the above field components and integration carried out over the range of $\mathrm{k}$ values from $-\infty$ to $+\infty$, with $\mathrm{g}(\mathrm{k})$ as :

$\underset{(4)}{\mathrm{g}(\mathrm{k})}=\left[\begin{array}{llllllllllllll} & 1 & / & (\sigma & (2 \pi) & 0 . & 5 & )\end{array}\right] \cdot \exp \left[\begin{array}{llllll}- & \left(\mathrm{k}-\mathrm{k}_{0}\right)^{2} & / & (2 & \sigma & 2\end{array}\right)$ Another complementary set of EM fields denoted by $(\mathbf{T E})_{2}$ is obtainable from the field set (3) by interchange of the $\cos (\theta)$ and $\sin (\theta)$ factors. In the present analysis, to begin with for simplicity, the order $n=0$ is considered for numerical evaluations. This then is taken as a representation of a photon described through its EM field set. The energy density of the EM field for the TE mode at any coordinate point $(s, \phi, z)$ at any time $t$ is then $u$, given by :

$\mathrm{u}=\left(\mathrm{B}_{\mathrm{Z}}{ }^{2}+\mathrm{B}_{\mathrm{s}}{ }^{2}+\mathrm{B}_{\phi}{ }^{2}+\mathrm{E}_{\mathrm{s}}{ }^{2}+\mathrm{E}_{\phi}{ }^{2}\right) /(8 \pi)$

(5)

Further integration of this energy density $u$ is carried out over the complete range of $s$ from 0 to $R$ and for $\phi$ from 0 to $2 \pi$, so that study of variation of energy density along the $z$ axis can be carried out. The energy densities obtained for the field sets $(\mathbf{T E})_{1}$ and $(\mathbf{T E})_{2}$, are given symbols $U_{Z_{1}}$ and $U_{Z_{2}}$, and average of these energy densities is then written as $U_{Z}$. For $n=1$ and higher orders the values of $U_{Z_{1}}$ and $U_{Z_{2}}$ are found to be equal and have the same analytical form as for the $U_{Z}$ obtained for the $n=0$ order. Thus, in general

$U_{z}=A^{2} \cdot\left\{f_{1}(z, t)+f_{2}(z, t)\right\} /\left(16 \pi^{2}\right) ; \quad$ where (6)

$\mathrm{f}_{1}(\mathrm{z}, \mathrm{t})=\left\{\int \cos (\theta)[\mathrm{g}(\mathrm{k})]^{0.5} \mathrm{dk}\right\}^{2}+\left\{\int(\mathrm{k} / \gamma) \sin (\theta)[\mathrm{g}(\mathrm{k})]^{0.5} \mathrm{dk}\right\}^{2}+\left\{\int[\omega /(\mathrm{c} \gamma)] \sin (\theta)[\mathrm{g}(\mathrm{k})]^{0.5} \mathrm{dk}\right.$ \}$^{2} ; \mathrm{f}_{2}(\mathrm{z}, \mathrm{t})=\left\{\int \sin (\theta)[\mathrm{g}(\mathrm{k})]^{0.5} \mathrm{dk}\right\}^{2}+\left\{\int(\mathrm{k} / \gamma) \cos (\theta)[\mathrm{g}(\mathrm{k})]^{0.5} \mathrm{dk}\right\}^{2}+\left\{\int[\omega /(\mathrm{c} \gamma)] \cos (\theta)\right.$ $\left.[\mathrm{g}(\mathrm{k})]^{0.5} \mathrm{dk}\right\}^{2}$;

with $\theta=(k z-\omega t)$. Further, integration of the $U_{z}$ over $z$ from $-\infty$ to $+\infty$ gives the energy $U$ of the photon, upon also completing the $\mathrm{k}$ integration as:

$\mathrm{U}=\mathrm{A}^{2}\left[\mathrm{k}_{0}{ }^{2}+\gamma^{2}+\sigma^{2}\right] \quad\left[\begin{array}{lll}4 \pi \gamma^{2} & ]\end{array}\right.$ (7)

Computational Results and Application to Laser Beams and Massive Particles:

Numerical evaluation of $U_{z}$ for the TE mode is done from equation (6), with value of $c$ taken as 1 and amplitude $A$ also taken as 1, for ease of calculation. The photon parameters are taken as:

$\mathrm{k}_{0}=\pi, \gamma=\pi, \sigma=0.1 \pi$. Figure 1 shows the variation of $\mathrm{U}_{\mathrm{z}}$ with $\mathrm{z}$ at $\mathrm{t}=0$. It is seen from the figure that the maxima of energy density of the EM field is located near $z=0$. Figure 2 shows the variation of $U_{z}$ with $z$ at times $t=0,5,10,15$, and 20 seconds (as series 1, 2, 3, 4 and 5 respectively). The location of the peak in energy density $U_{z}$ permits identification of the photon location $z(t)$ at different times $t$. Figure 3 shows the variation of photon location $z(t)$ with time $t$ for two values of $\gamma$ as $\pi$ and $2 \pi$ (as series 1 and 2)and it is seen that the velocity $v_{g}=d z(t) / d t$ of the photon comes out to be less than the unity value taken for $c$. By studying other examples with different $\mathrm{k}_{0}$ and $\gamma$ values it is found that the photon velocity 
fits very well the group velocity formula: $v_{g}=c k 0 /\left[k_{0}^{2}+\gamma{ }^{2}\right]$. 5 (8) When the parameter $\gamma$ is zero, the field solution given by field set (3) can be reduced to that for plane waves, and in that case our value of $v_{g}$ also becomes equal to $c$. Thus it is stated that for photons having narrow dimensions in the direction transverse to the direction of propagation, such as for laser beams of light, the velocity of the laser photons in vacuum can be smaller than the standard velocity of light in vacuum, depending upon how narrow is the laser beam of light. For describing the lateral size of the photon in the case of the above studied example $\left(\mathrm{k}_{0}=\pi, \gamma=\pi, \sigma=0.1 \pi\right.$ ) the energy density $u$ ( averaged over the pair of complementary fields ) for the $n=0$ order is shown with the radial coordinate $s$ in figure 4 at time $\mathrm{t}=0$, at position $\mathrm{z}=0$; and at time $\mathrm{t}=40$ seconds at position $\mathrm{z}=\mathrm{v}_{\mathrm{g}} \mathrm{t}=28.284$ ( as series 1 and 2 respectively). It is seen that the graphs for the two times almost overlap each other in radial dependence. The transverse radial width of the central peak in the energy density of the photon is found to be nearly $3.832 / \gamma$ where the factor 3.832 arises from the first zero of Bessel function $J_{1}$. As an example, for ruby laser light ${ }^{2}$ of wavelength 0.6943 micron, with the laser beam radius as $1 \mathrm{~mm} ; \mathrm{k}_{0}=9.049669$ micron $^{-1}, \gamma=3.832 \mathrm{~mm}^{-1}$, which gives $v_{g}=0.9999999103 \mathrm{c}$. Thus experimental measurements involving narrow laser beams of light should correct for this reduction in light velocity. It is further noted from figures 2 and 4 that with progression of time, the peak height of the energy density slowly decreases and its width increases along the $z$ axis, due to the individual $(k, \gamma)$ components in the wave packet having different phase velocities. There is no transverse spreading of the wave packet un-like that for laser beams ${ }^{2}$ of light, while the longitudinal spreading is like that for quantum mechanical ${ }^{3}$ spreading of particle wave packets. Thus narrow lateral width photons travel at slower than the standard velocity of light, and also have spreading in size, and on both these counts behave like matter particles. If photon energy $U$ is quantized as $\hbar \omega_{0}$ and photon momentum $\mathrm{p}$ as $\hbar \mathrm{k}_{0}$, then equation $\left(\omega_{0} / \mathrm{c}\right)^{2}=\mathrm{k}_{0}{ }^{2}+\gamma^{2}$ can be transformed to $: U^{2}=c^{2} p^{2}+m_{0}^{2} c^{4}$

with $\quad m_{0}$

$\mathrm{m}_{0} \quad=$

$\hbar$

$\gamma$

c

(9)

giving a finite rest mass to the photon depending upon its transverse size, while at the same time providing the relativistic energy-momentum relationship of special theory of relativity ${ }^{3 \& 4}$

\section{Application to Electrons and Protons:}

This analysis is further applied to electrons , considering them to be wave packets of Electromagnetic field. Taking the rest mass of the electron as $m_{e}$ and putting it equal to $\hbar \gamma / c$ from equation (9), the value of the Bessel parameter $\gamma$ is obtained as $2.5896 \times 10^{10} \mathrm{~cm}^{-1}$. Further to consider the electron in motion a typical value of $k_{0}=10000 \pi$ is chosen, and $\sigma$ is taken as $10 \pi$ for an example. Figure 5 shows the numerically evaluated energy density $\mathrm{Uz}$ evaluated from equation (6) as a function of axial coordinate $z$ at times $t=0.0 \mathrm{sec}$, $0.00001 \mathrm{sec}, 0.00002 \mathrm{sec}$ and $0.00003 \mathrm{sec}$. The position of the peak in Uz at time $\mathrm{t}=$ 0.00001 second is found to be at $z=0.365 \mathrm{~cm}$, which agrees very well with the group velocity $\mathrm{v}_{\mathrm{g}}=36369.5 \mathrm{~cm} / \mathrm{sec}$ given by equation (8) with the chosen values of $\mathrm{k}_{0}$ and $\gamma$. For comparison the plane wave beam of light in time $t=0.00001 \mathrm{sec}$ would have traveled $299792 \mathrm{~cm}$. Thus the electron does behave like a slow photon as described above using electro-magnetic field for its description. Further, an evaluation of particle velocity from $\hbar \mathrm{k}_{0} /$ $m_{e}$ also gives its velocity agreeing exactly with that from equation (8). The radial dependence of the energy density $u$ with radial coordinate $s$ is shown for the $n=0$ order considered in figure 6 at time $\mathrm{t}=0 \mathrm{sec}$ at $\mathrm{z}=0 \mathrm{~cm}$; and at time $\mathrm{t}=0.00001 \mathrm{sec}$ at $\mathrm{z}=0.365$ $\mathrm{cm}$ as (series 1 and 2 ), and it is seen that it falls very rapidly with radius near $\mathrm{s}=0$, but at higher $s$ values it falls quite slowly as $1 / \mathrm{s}$, since it is arising due to square of the Bessel functions. The long range slow fall of the energy density $u$ with radius should permit the electron to have long range interaction with other electrons, as is required for Coulomb 
interaction ${ }^{1}$ between charged particles. This description of the electron as a slow photon allows it to be transversely compact ( localized) and still be de-localized enough for long range interactions in transverse directions. Results for motion of protons with time shall be similar to those shown in figures 5 and 6 for the electron, with the mass of the proton $m_{p}$ to be used in place of mass $m_{e}$ of the electron in the numerical evaluations.

\section{Linear Momentum:}

The linear momentum density ${ }^{1}$ of the Electro-magnetic field is given by (ExB) / $(4 \pi \mathrm{C})$. Using one TE mode of EM field given by $(T E)_{1}$, and integrating the momentum density over the complete volume, including integration over the range of $k$ values, one obtains the $z$ component of the total momentum of the field ( now the particle ) as:

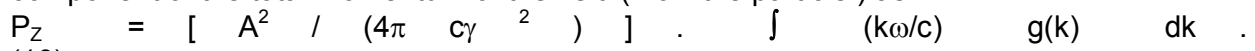

(10)

It is easily verified analytically that this result applies equally well for TM and TE modes, for each individual order $\mathrm{n}$ of the Bessel function. For the case of non-zero rest mass particles like electron the value of $\gamma$ is much larger than $k_{0}$ and the value of $P_{Z}$ from equation (10) reduces to

$P_{z}=A^{2} k_{0} /(4 \pi c \gamma)$; which is like what it should be for a particle of rest mass $m_{0}=\hbar \gamma / c$, rest mass energy as $U_{0}=A^{2} /(4 \pi)$ and velocity $v_{g}=c k_{0} / \gamma$ from equations (7), (8) and (9). For the other case where $k_{0}$ is much larger than $\gamma$, such as for laser beam of light, the value of $P_{z}$ from equation (10) reduces to: $P_{z}=A^{2}\left[k_{0}{ }^{2}+\sigma^{2}\right] /\left[4 \pi c \gamma^{2}\right]$; which on comparison with $U$ from equation (7) shows that $P_{Z}=U / c$ as it should be for normal photons. Thus our description of Slow Photons in Vacuum is capable of explaining several aspects of light as well as of particles having non-zero rest mass like Electrons. A hypotheses is made at this stage of the paper that the Elementary Particles like Electrons and Protons are actually wave packets of Electro-Magnetic field and these wave packets need to be further investigated to fully establish the identity of the fundamental particles.

\section{Quantization:}

The wave packets are now quantized, so that the amplitude A of the EM fields can be specified, and the quantization condition is defined through the energy of the wave packet , equally well for both TM and TE modes as :
$\mathrm{U}=\mathrm{A}^{2}\left[\mathrm{k}_{0}^{2}+\gamma^{2}+\sigma^{2}\right]$
/ $\left[4 \pi \gamma^{2}\right]$
$=$
$\hbar$ C $\left[k_{0}{ }^{2}+\gamma^{2}+\sigma^{2}\right] \quad$ o. $\quad 5$

(11)

It is seen that in the limit of $\mathrm{k}_{0}$ much larger than $\gamma$ and $\sigma, \mathrm{U}$ becomes equal to $\hbar c \mathrm{k}_{0}$ with $\omega$ also becoming equal to $\mathrm{ck}_{0}$; and in the limit of $\gamma$ much larger than $\mathrm{k}_{0}$ and $\sigma, \mathrm{U}$ becomes equal to $\hbar c \gamma$ with $\omega$ also becoming equal to $c \gamma$. The quantization condition (11) thus leads to :

$\mathrm{A}^{2}=4 \pi \quad \hbar \quad \mathrm{c} \quad \gamma^{2} \quad \begin{array}{lllllll}\left.\mathrm{k}_{0}{ }^{2}+\gamma^{2}+\sigma^{2}\right] & - & 0 & 5\end{array}$

(12)

The linear momentum in the $\mathrm{z}$ direction, under the above given quantization condition becomes:

$\mathrm{P}_{\mathrm{z}}=\hbar \quad\left[\mathrm{k}_{0}{ }^{2}+\gamma^{2}+\sigma^{2}\right]-\begin{array}{ccccccccc}0 & 5 & 5 & \mathrm{k} & {\left[\mathrm{k}^{2}+\gamma^{2}\right]} & 0 . & 5 & \mathrm{~g}(\mathrm{k}) & \mathrm{dk}\end{array}$

(13)

In the limit of $k_{0}$ large compared to $\gamma$ and $\sigma, P_{z}$ becomes $\hbar k_{0}$ and $U$ becomes $\hbar c k_{0}$, leading to $P_{z}$ equal to $U / C$ as is normally required for light photons. Further, in the limit of $\gamma$ large compared to $\mathrm{k}_{0}$ and $\sigma, \mathrm{P}_{\mathrm{Z}}$ again becomes $\hbar \mathrm{k}_{0}$ while $U$ becomes $\hbar c \gamma$ which is like that for a particle of rest mass $\hbar \gamma / c$ as already noted above.

Angular Momentum:

The EM field set given by the (TE) 1 mode given by equations (3) for any order $n$ can be used for evaluation of the orbital angular momentum density $\mathbf{r} \mathbf{x}($ ExB $) /(4 \pi \mathrm{c})$ of the EM field for any order $n$. The $z$ component of the angular momentum density of the EM field, in cylindrical coordinates is $s\left(E_{z} B_{S}-E_{S} B_{Z}\right) /(4 \pi c)$ which for TM modes becomes $s E_{z}$ 
$B_{S} /(4 \pi c)$ and for TE modes becomes - $s E_{S} B_{Z} /(4 \pi c)$ for any order $n$. Integration over the complete volume then gives the $z$ component of Angular Momentum $L_{z}$ as :

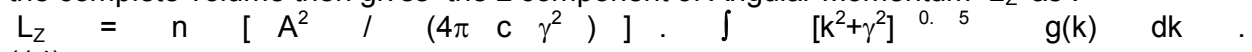
(14)

for each of the $(T E)_{1}$ and (TE $)_{2}$ modes, for any order $n$, with the value of $L_{z}$ being same for TM modes as well. Upon putting the value of $A^{2}$ from equation (12) this value of $L_{z}$ becomes :

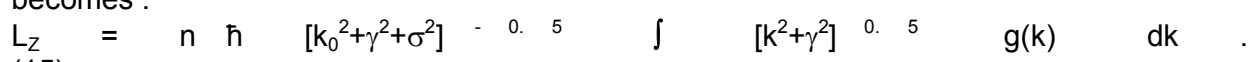
(15)

It is seen that in the limit of $k_{0}$ much larger than $\gamma$ and $\sigma$ the $L_{z}$ becomes equal to $n \hbar$, and in the limit of $\gamma$ much larger than $k_{0}$ and $\sigma$ the $L_{z}$ again becomes equal to $n \hbar$. Thus it is seen that for both normal light photons and for electron particle like photon wave packets the $z$ component of angular momentum of the EM field is $n \hbar$. As the Bessel functions $J_{n}$ can have positive and negative values of $n$, the $z$ component of angular momentum of any given individual TE (or TM) mode can have a value $n \hbar$ or $-n \hbar$. Thus for a given order of magnitude $|n|$ only two possible values of $L_{z}$ are allowed as $n \hbar$ or $-n \hbar$, and not $(2 n$ +1 ) values. The two spin states of the electron, with spin as $\pm 1 / 2$ as introduced by the description of the electron through the Dirac equation ${ }^{3}$ now appear simply as only two possible azimuthal dependences of the wave function, associated with Bessel function of any integer order except for the zeroth order.

It is thus shown that under different values of wave parameters $\mathbf{k}_{0}, \gamma$ and Bessel order $\mathrm{n}$ these slow EM wave packets in vacuum can appear like light photons as well as like electrons and protons, with regard to their mechanical features of velocity, energy, rest mass, linear momentum and angular momentum.

\section{References}

1) J. D .Jackson, "Classical Electrodynamics" IIIrd Edition, Chapter 7, John Wiley \& Sons, Inc., USA,1999.

2) Ajoy Ghatak and K.Thyagarajan, "Optical Electronics", Chapter 5 and 9 , Cambridge University Press, UK, 1991.

3) Leonard I. Schiff, “Quantum Mechanics” IIIrd Edition, Chapters 3 and 13, Mc-Graw Hill Book Company, USA,1968.

4) Albert Einstein, "The Meaning of Relativity" VIth Edition, Chapter 2 , Princeton University Press, USA, 1956.

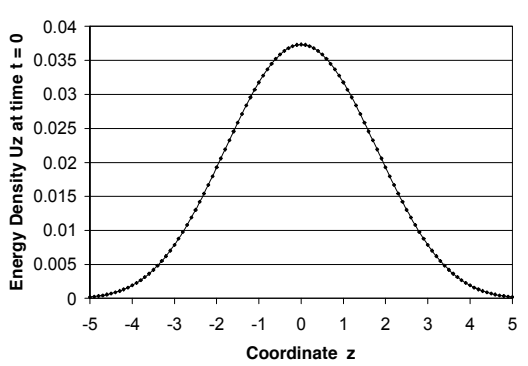

Figure 1.

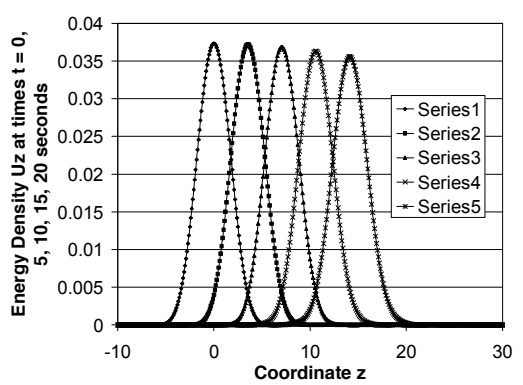

Figure 2. 


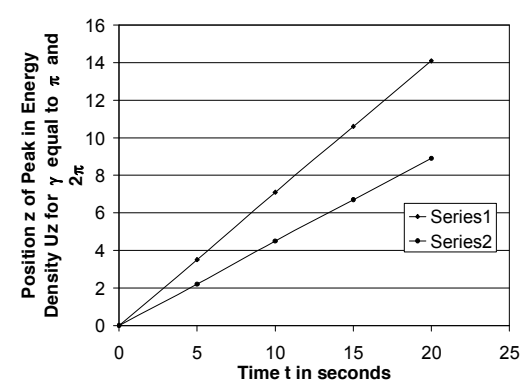

Figure 3.

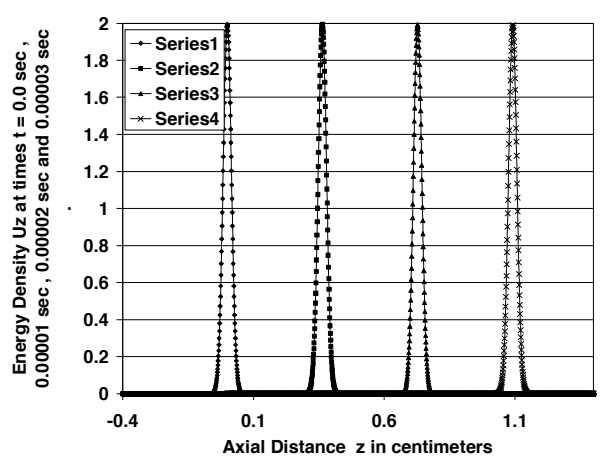

Figure 5.

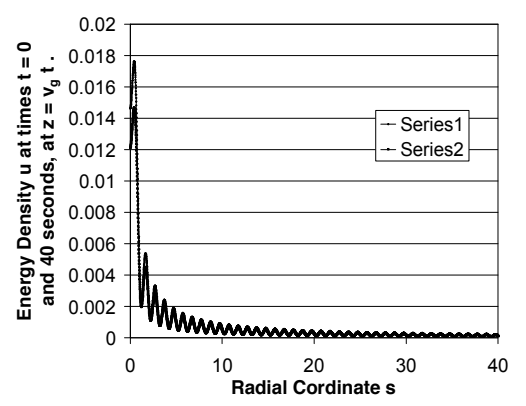

Figure 4.

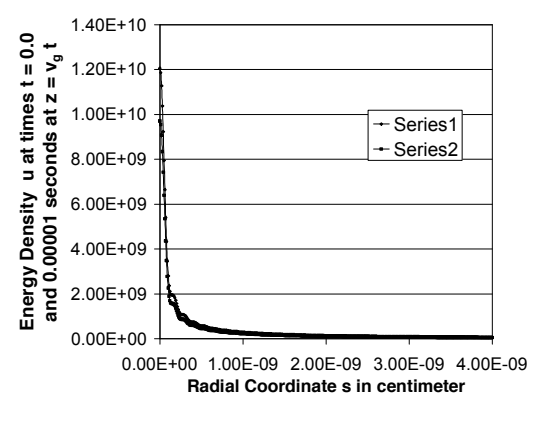

Figure 6 . 